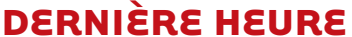

\section{Mécanisme d'inhibition de la production hépatique de glucose par la metformine}

Un nouveau concept pour un ancien remède

Marc Foretz, Benoît Viollet

La metformine, un antidiabétique moderne issu du passé

La metformine (1,1-diméthylbiguanide hydrochloride) est un antidiabétique oral de la famille des biguanides. Les biguanides tirent leur origine d'un alcaloïde, la galégine, issue du galéga officinal (Galega officinalis), une plante légumineuse utilisée dans l'Europe médiévale pour soigner la polyurie chez des sujets diabétiques. La metformine est commercialisée en France depuis 1979 sous l'appellation évocatrice de Glucophage ${ }^{\circledR}$ mais n'est adoptée que depuis 1990 par les États-Unis. La metformine est utilisée avec succès depuis plus de 40 ans dans le traitement du diabète de type 2. C'est le traitement de première intention chez les diabétiques de type 2, en particulier chez les patients en surpoids ou obèses, pour son efficacité, sa faible capacité à provoquer des hypoglycémies et l'absence de prise de poids. La metformine exerce des effets bénéfiques sur le métabolisme énergétique et le système cardiovasculaire. Le foie joue un rôle central dans les effets métaboliques de la metformine: la principale action de cette molécule est en effet d'améliorer l'hyperglycémie par inhibition de la gluconéogenèse hépatique [1].

\section{Action transcriptionnelle} de la metformine : un mécanisme d'action privilégié mais non prouvé Malgré I'utilisation généralisée et l'efficacité incontestée de la metfor- mine dans le traitement du diabète de type 2, son mécanisme d'action est mal compris. Un certain nombre de mécanismes moléculaires ont été proposés pour expliquer ses effets dans l'inhibition de la gluconéogenèse hépatique. Depuis quelques années, la littérature privilégie une action transcriptionnelle de la metformine.

L'entrée de la metformine dans la cellule se fait via le transporteur de cations organiques 0CTl (organic cation transporter 1). L'expression importante de OCTl dans les hépatocytes confère au foie le statut de tissu cible de la metformine. Ainsi, la délétion de OCTl dans des hépatocytes de souris entraîne une altération de l'effet inhibiteur de la metformine sur la gluconéogenèse [2]. $\varepsilon$ n 2001, Zhou et al. ont montré que la metformine active I'AMP-activated protein kinase (AMPK) dans des hépatocytes et dans le muscle, ce qui, selon eux, fournit une explication globale des effets pléiotropes favorables de ce médicament; ils ne proposaient toutefois pas de mécanisme pour cette activation [3]. L'AMPK est une sérine thréonine kinase exprimée dans toutes les cellules eucaryotes. C'est un hétérotrimère composé d'une sous-unité catalytique $\alpha$ et de deux sous-unités régulatrices $\beta$ et $\gamma$. L'AMPK est activée par phosphorylation de la thréonine 172 de la sous-unité catalytique $\alpha$ par une AMPK kinase, en particulier le suppresseur de tumeur LKBI [4]. L'AMPK est un
Institut Cochin, Université Paris Descartes, CNRS (UMR 8104), Inserm, U1016,

Institut Cochin, Département endocrinologie, métabolisme et cancer,

24, rue du Faubourg Saint-Jacques,

75014 Paris, France.

marc.foretz@inserm.fr

senseur métabolique sensible au niveau énergétique de la cellule qui permet d'ajuster en permanence, et au plus près, les besoins et disponibilités énergétiques de la cellule. Elle est activée en réponse à une augmentation du rapport intracellulaire AMP/ATP induite par un stress métabolique comme l'exercice musculaire, l'hypoxie ou le jeûne [4]. Une fois activée, l'AMPK inhibe les voies métaboliques anaboliques consommatrices d'ATP et active les voies cataboliques productrices d'ATP afin de rétablir le rapport AMP/ATP intracellulaire. Par exemple, I'AMPK augmente l'oxydation des acides gras et inhibe la lipogenèse dans le foie [4].

Récemment, on a attribué l'inhibition de la production de glucose par la metformine à l'activation de I'AMPK via l'inhibition de l'expression des gènes de la gluconéogenèse. Selon ce schéma, I'AMPK, activée par $L K B I$ en réponse à la metformine, inhibe en le phosphorylant le co-activateur transcriptionnel TORC2 (transcriptional co-activator transducer of regulated CREB activity 2). En effet, la phosphorylation de TORC2 entraîne sa translocation dans le cytoplasme; de ce fait, le facteur de transcription PGC- $1 \alpha$ (PPAR $\gamma$ coactivator $1 \alpha$ ), un acteur central dans l'activation du programme gluconéogénique $[5,6]$, n'est plus exprimé, ce qui aboutit à l'absence d'expression des gènes de la gluconéogenèse.

Toutefois, ce mécanisme transcriptionnel ne permet pas d'expliquer l'inhi- 


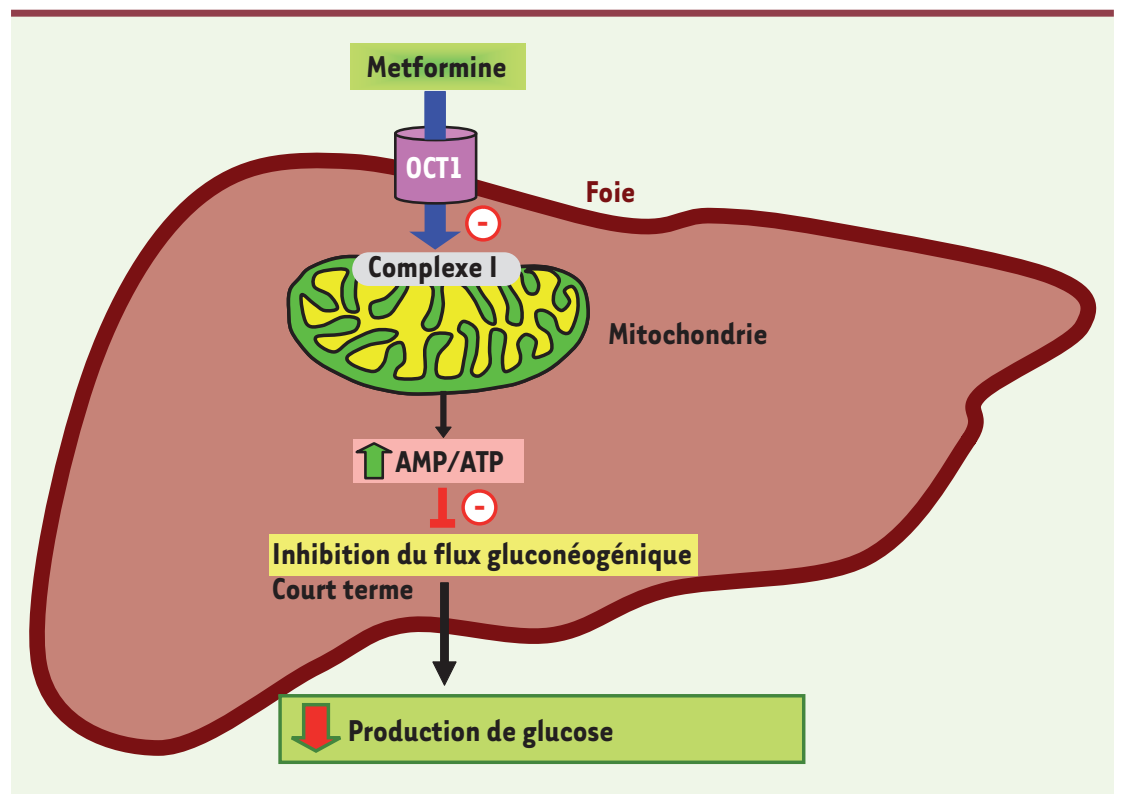

bition immédiate de la production de glucose qu'engendre la metformine. En utilisant un modèle murin délété de I'AMPK dans le foie, nous avons réexaminé l'action de la metformine sur la production hépatique de glucose [7].

\section{Hypothèse alternative :}

la metformine agit indépendamment de la voie LKB1-AMPK

\section{La metformine inhibe la production de glucose indépendamment d'un effet transcriptionnel}

Le mécanisme décrit précédemment prédit que l'inhibition transcriptionnelle qu'induit la metformine sur les gènes de la gluconéogenèse s'exerce en amont de PGC-l $\alpha$. Cependant, nous avons observé que la metformine est toujours capable d'inhiber la production hépatique de glucose dans des hépatocytes en culture primaire même si l'on y force l'expression des gènes de la gluconéogenèse par la surexpression de PGC-1 $\alpha$ [7]. La metformine est donc capable d'inhiber la gluconéogenèse indépendamment d'un effet transcriptionnel.

\section{L'AMPK n'a pas d'effet direct sur la gluconéogenèse}

Notre étude montre que, contrairement à la délétion hépatique de LKBI qui s'accompagne d'une hyperglycémie et d'une augmentation de l'expression des gènes de la gluconéogenèse [6], la délétion de l'AMPK dans le foie n'affecte pas la glycémie chez la souris [7]. De même, la production de glucose et l'expression des gènes de la gluconéogenèse dans des hépatocytes dépourvus d'AMPK ne sont pas altérées en condition basale ou après stimulation par l'AMPc. De plus, l'activation spécifique de l'AMPK avec un activateur direct (A-769662) qui ne modifie pas le rapport intracellulaire AMP/ATP n'a aucun effet sur la production de glucose et sur l'expression des gènes de la gluconéogenèse dans des hépatocytes [7]. Nous concluons que la modulation de l'activité de l'AMPK dans le foie n'a pas de conséquence directe sur la régulation de la gluconéogenèse.

\section{La metformine inhibe}

la gluconéogenèse indépendamment de la voie LKB1-AMPK en diminuant la charge énergétique du foie

De manière inattendue, nous avons observé que l'inhibition de la production de glucose par la metformine persiste dans des hépatocytes même en l'absence de l'AMPK ou de LKBI et qu'elle est plus marquée que dans des hépatocytes contrôles [7]. Il est important d'évoquer à ce stade les travaux longtemps

Figure 1. La metformine inhibe à court terme la production de glucose hépatique en induisant une diminution du statut énergétique du foie. La metformine entre dans l'hépatocyte via le transporteur OCTl. Dans la cellule, la metformine inhibe partiellement le complexe I de la chaîne respiratoire mitochondriale. Cette inhibition entraîne une augmentation du rapport AMP/ATP, modérée mais suffisante pour réduire le flux de la gluconéogenèse. Cet effet à court terme est indépendant d'un mécanisme transcriptionnel et de la voie LKBI-AMPK.

passés inaperçus de l'équipe de Xavier Leverve à Grenoble sur la metformine. Ce groupe a montré pour la première fois que la metformine inhibe le complexe I (NADH : ubiquinone oxydoréductase) de la chaîne respiratoire mitochondriale de manière spécifique et non toxique, avec pour conséquence une augmentation modérée du rapport AMP/ATP dans des hépatocytes [8]. Ces observations ont été confirmées la même année par un groupe anglais [9]. Ces résultats importants offrent une explication à l'activation de l'AMPK par la metformine. Ainsi, le complexe I de la chaîne respiratoire est la cible primaire de la metformine et I'AMPK est activée indirectement via l'augmentation de la concentration de l'AMP intracellulaire. En outre, cette action sur la mitochondrie pourrait permettre aussi d'expliquer la rapidité de l'inhibition de la gluconéogenèse par la metformine. $\varepsilon n$ effet, la gluconéogenèse est une voie métabolique très consommatrice d'énergie puisque qu'elle requiert 4 ATP et 1 GTP par molécule de glucose formée. Par conséquent, nous avons formulé I'hypothèse selon laquelle la metformine inhibe le flux gluconéogénique en diminuant la synthèse d'ATP. Ainsi, nous avons pu montrer que la metformine à des concentrations thérapeutiques 


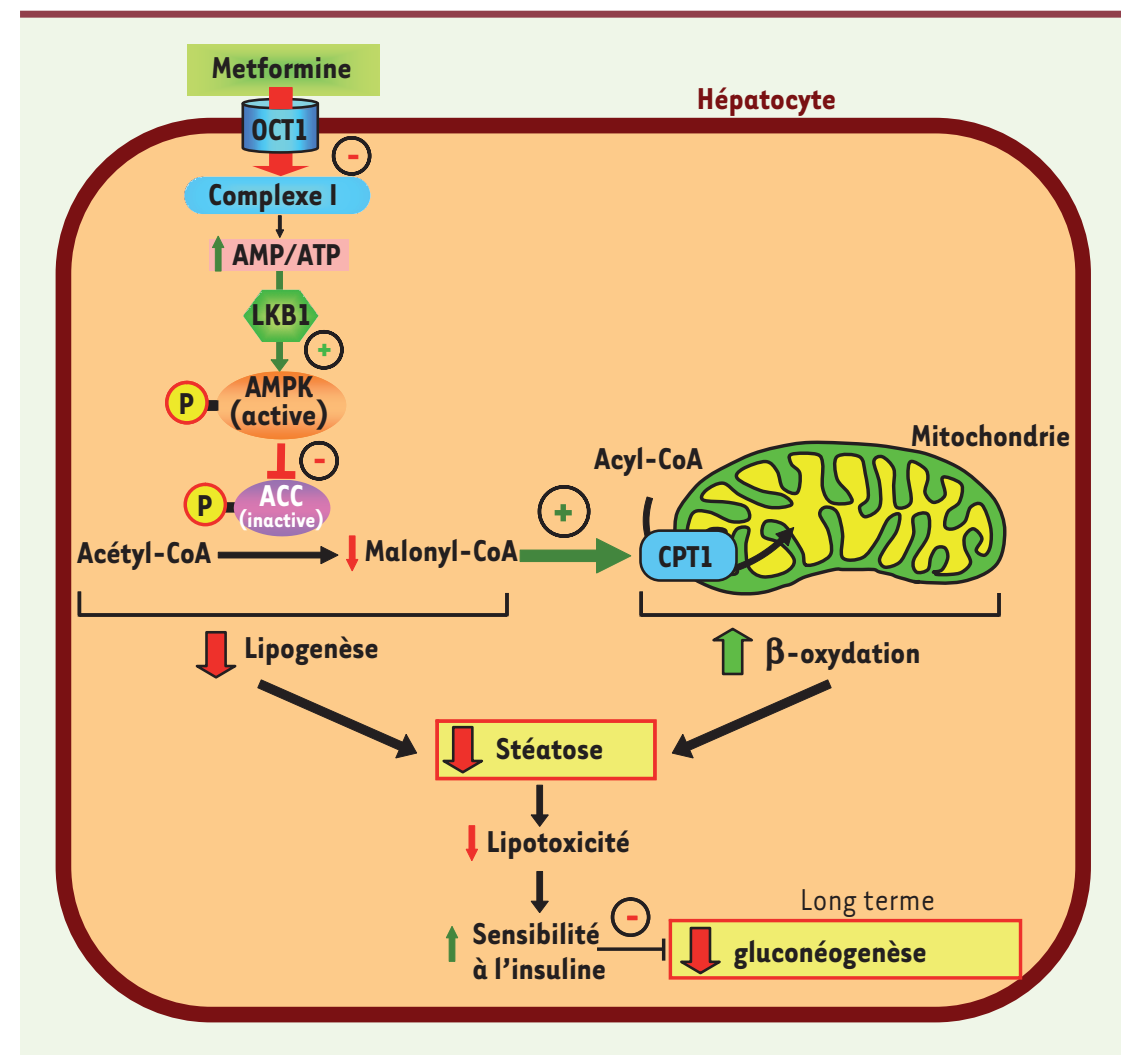

augmente le rapport AMP/ATP in vivo dans le foie et dans des hépatocytes isolés de souris [7]. L'effet inhibiteur de la metformine sur les niveaux intracellulaires d'ATP est accentué dans des hépatocytes dépourvus d'AMPK, révélant le rôle de senseur énergétique de I'AMPK [7]. Finalement, nous montrons que l'inhibition de la production de glucose par la metformine est étroitement corrélée avec le contenu intracellulaire en ATP [7]. De plus, l'augmentation des concentrations intracellulaires d'AMP inhibe de manière allostérique la fructose-1,6-biphosphatase, une enzymeclé de la gluconéogenèse, contribuant à l'inhibition de la production de glucose. Ainsi, la metformine inhibe le flux gluconéogénique en diminuant la synthèse d'ATP indépendamment de la voie LKBlAMPK (Figure 1).

\section{Perspectives:}

I'AMPK n'a pas dit son dernier mot Bien que nos résultats montrent que I'AMPK n'a pas d'effet direct sur la production de glucose, nous pensons qu'à long terme une partie des effets de la metformine sur la gluconéogenèse hépatique pourraient dépendre de I'AMPK [1]. Plusieurs études suggèrent que la metformine améliore la stéatose hépatique, une accumulation de triglycérides fréquemment associée au diabète de type 2 [10]. De fait, la metformine active I'AMPK dans le foie. Indiscutablement, I'AMPK exerce une action majeure sur le métabolisme lipidique en inhibant la lipogenèse et en stimulant la $\beta$-oxydation. L'AMPK phosphoryle et inhibe en effet les deux enzymes-clés qui contrôlent la synthèse du cholestérol et des acides gras : la HMGCoA réductase et l'acétyl-CoA carboxylase ( $A C C$ ). L'inactivation de l'ACC par I'AMPK conduit à une diminution de la concentration du malonyl-CoA et à une augmentation de l'activité de la CPT-1 (carnitine palmitoyltransférase 1 ) et de l'oxydation des acides gras (Figure 2). En conséquence, l'activation de l'AMPK par la metformine est susceptible de réduire à long terme l'accumulation de lipides dans le foie. La diminution du contenu
Figure 2. La metformine est susceptible d'inhiber à long terme la gluconéogenèse en améliorant la stéatose hépatique par l'activation de l'AMPK. Dans l'hépatocyte, la metformine active de manière indirecte l'AMPK. L'augmentation du rapport AMP/ATP provoque I'activation de l'AMPK par phosphorylation via LKBI. L'AMPK inhibe par phosphorylation l'acétyl-CoA carboxylase ( $A C C)$, une enzyme qui contrôle la lipogenèse. L'inactivation de l'ACC conduit à une diminution de la concentration du malonyl-CoA et à une augmentation de l'activité de la carnitine palmitoyl-transférase 1 (CPT-1) et de l'oxydation des acides gras. À long terme, ce mécanisme pourrait améliorer la stéatose hépatique souvent associée au diabète de type 2 . En conséquence, la diminution de la lipotoxicité dans l'hépatocyte pourra rétablir la sensibilité à l'insuline et permettre l'inhibition de la gluconéogenèse.

lipidique dans les hépatocytes limite le phénomène de lipotoxicité et contribue à améliorer la transduction du signal induit par l'insuline (Figure 2). Dans un contexte de diabète de type 2 , cet effet sur la sensibilité à l'insuline pourra de manière indirecte participer à l'amélioration de l'hyperglycémie en restaurant l'inhibition de la gluconéogenèse hépatique par l'insuline [1] (Figure 2).

\section{Conclusion}

Nos récents travaux montrent que la metformine inhibe la production de glucose hépatique à court terme en réduisant le flux gluconéogénique par une action purement énergétique indépendamment d'un effet transcriptionnel et de l'axe LKBI-AMPK. Cet effet résulte d'une inhibition modérée du complexe I de la chaîne respiratoire mitochondriale qui entraîne une augmentation du rapport AMP/ATP, certes faible, mais suffisante pour réduire le flux de la gluconéogenèse (Figure 1). Ce mécanisme impliquant une diminution mesurée du statut énergétique du foie fait de la 
chaîne respiratoire mitochondriale une cible thérapeutique du diabète de type 2 pour le développement de molécules inhibant avec parcimonie son activité. $\diamond$ Mechanism of inhibition of hepatic glucose production by metformin: a new concept for an old remedy

\section{REMERCIEMENTS}

Ce travail est soutenu par la Commission européenne (LSHM-CT-2004-005272/exgenesis), l'Association pour l'étude des diabètes et des maladies métaboliques (ALFEDIAM), le Programme national de recherche sur le diabète (PNRD), l'Association de recherche sur le diabète (ARD), l'Institut Benjamin Delessert, l'Association pour la recherche sur le diabète, l'insuffisance cérébrale et le cancer (AREDIC) et l'Institut Appert.

\section{CONFLIT D'INTÉRÊTS}

Les auteurs déclarent n'avoir aucun conflit d'intérêts concernant les données publiées dans cet article.

\section{RÉFÉRENCES}

1. Foretz M, Viollet B. Mécanisme d'action hépatique de la metformine dans le diabète de type 2. Med Mal Metab 2009; $3: 48-54$.

2. Shu Y, Sheardown SA, Brown C, et al. Effect of genetic variation in the organic cation transporter 1 (OCT1) on metformin action. J Clin Invest 2007 ; $117: 1422-31$.

3. Zhou G, Myers R, Li Y, et al. Role of AMP-activated protein kinase in mechanism of metformin action. J Clin Invest 2001 ; 108 : 1167-74.

4. Foretz M, Taleux N, Guigas B, et al. Régulation du métabolisme énergétique par l'AMPK : une nouvelle voie thérapeutique pour le traitement des maladies métaboliques et cardiaques. Med Sci (Paris) 2006 ; 22: 381-8.

5. Foretz M, Guigas B, Viollet B. Du cancer au traitement du diabète : le suppresseur de tumeur LKBl comme nouvelle cible pharmacologique. Med Sci (Paris) $2006 ; 22: 348-50$.
6. Shaw RJ, Lamia KA, Vasquez D, et al. The kinase LKBl mediates glucose homeostasis in liver and therapeutic effects of metformin. Science 2005; $310: 1642-6$.

7. Foretz M, Hébrard S, Leclerc J, et al. Metformin inhibits hepatic gluconeogenesis in mice independently of the LKB1/AMPK pathway via a decrease in hepatic energy state. J Clin Invest 2010 ; 120 (sous presse)

8. $\varepsilon l$-Mir MY, Nogueira V, Fontaine $\varepsilon$, et al. Dimethylbiguanide inhibits cell respiration via an indirect effect targeted on the respiratory chain complex I.J Biol Chem $2000 ; 275: 223-8$.

9. Owen MR, Doran $\varepsilon$, Halestrap AP. Evidence that metformin exerts its anti-diabetic effects through inhibition of complex 1 of the mitochondrial respiratory chain. Biochem J $2000 ; 348: 607-14$.

10. Lin HZ, Yang SQ, Chuckaree C, et al. Metformin reverses fatty liver disease in obese, leptindeficient mice. Nat Med $2000 ; 6: 998-1003$.
TIRÉS À PART

M. Foretz 